УДК 539.2

\title{
ЛОКАЛИЗОВАННЫЕ СОСТОЯНИЯ НА ГРАНИЦЕ ЛИНЕЙНОЙ И НЕЛИНЕЙНОЙ СРЕД
}

\author{
(C) 2017 С. Е. Савотченко \\ Белгородский государственный технологический университет имени В.Г. Шухова, \\ ул. Костюкова, 46, 308012 Белгород, Россия \\ e-mail:savotchenkose@mail.ru \\ Поступила в редакцию: 02.10.2017
}

\begin{abstract}
Аннотация. Предложена модель, описывающая особенности локализации возбуждений на границе раздела линейной и нелинейной сред. В основе модели лежат линейное и нелинейное уравнения Шредингера в полупространствах. Показано, что возможно существование нелинейных локализованных возбуждений нескольких типов. Структура и форма локализованных состояний определяется знаком ангармонизма взаимодействия в среде и интенсивностью взаимодействия возбуждений с дефектом. Установлено, что в сплошном спектре существуют квазилокальные состояния, монотонно затухающие в полупространстве с нелинейной средой при переходе через границу раздела.
\end{abstract}

Ключевые слова: нелинейное уравнение Шредингера, граница раздела сред, солитон, локализованные состояния, квазилокальные состояния, плотность состояний.

\section{ВВЕДЕНИЕ}

При разработке многих технических компонентов микроэлектроники и оптоэлектроники особое значение имеет информация о закономерностях локализации возбуждений вблизи границ раздела сред. Для реализации механизмов управления сложных электронных и оптических систем может представлять интерес построения контролируемых барьеров посредством границы раздела сред с различными физическими свойствами, в частности, с различными характеристиками ангармонизма межатомного взаимодействия. Принято называть нелинейной такую среду, в которой существенны анграмонические взаимодействия, а линейной ту, в которой межатомное взаимодействие является гармоническим. В результате при переходе через границу раздела таких сред возбуждения терпят различные преобразования, которые обусловлены характером взаимодействия этих возбуждений с границей как плоским дефектом кристаллической структуры. В [1] при описании локализации электромагнитных волн вблизи границ раздела нелинейной и линейной сред показана возможность существования нелинейных локализованных волн с несимметричным профилем, отличающихся от свободно распространяющихся солитонов и называемых нелинейными поверхностными волнами.
Для построения математических моделей взаимодействия возбуждений с дефектами в нелинейных средах широко применяется нелинейное уравнение Шредингера (НУШ) [2, 3]. Как было показано в [4], НУШ может описывать состояния полей различной физической природы: упругого, электрического и магнитного. Поэтому для описания новых эффектов, связанных с локализацией возбуждений различной физической природы вблизи дефектов, имеет смысл далее рассматривать математическую модель, использующую НУШ, которому подчиняется функция $\psi$, выступающая в роли огибающей комплексного поля компонент вектора намагниченности в легкоосном ферромагнетике, либо комплексной амплитуды упругого поля смещения сдвиговой волны в кубическом кристалле с плоским дефектом, либо комплексной функции из амплитуд компонент электрического поля в оптической нелинейной среде [5]. Тогда параметры в уравнениях будут иметь соответствующий физических смысл в рамках одной из трех указанных моделей.

В данной работе будут решены НУШ и получены энергетические уровни стационарных локализованных вблизи границы раздела линейной и нелинейной сред. Будет показано, что существует несимметричное состояние с энергией в полосе сплошного спектра линейных волн, представляющее собой стоячую волну в линейной среде и зату- 
хающую при удалении от границы раздела в нелинейной среде. Рассчитаны добавки к спектральной плотности таких состояний. Отдельно рассмотрены случаи, как положительной нелинейности, так и отрицательной.

\section{1. ФОРМУЛИРОВКА МОДЕЛИ}

Будем рассматривать процессы локализации возбуждений на границе раздела линейной и нелинейной сред на основе уравнения Шредингера:

$$
i \frac{\partial \psi}{\partial t}=-\frac{1}{2 m} \frac{\partial^{2} \psi}{\partial x^{2}}+\Omega(x) \psi-\gamma \theta(x)|\psi|^{2} \psi+U_{0} \delta(x) \psi,(1)
$$

где использована тета-функция Хевисайда:

$$
\theta(x)=\left\{\begin{array}{l}
0, x<0 \\
1, x>0
\end{array}\right.
$$

$m$ - эффективная масса возбуждений,

$$
\Omega(x)=\left\{\begin{array}{l}
\Omega_{1}, x<0 \\
\Omega_{2}, x>0
\end{array}\right.
$$

$\Omega_{1,2}-$ значения уровней дна энергетический зоны, $\gamma$-параметр нелинейности среды, расположенной справа от дефекта, $\delta(x)-\delta$-функция Дирака, $U_{0}-$ интенсивность взаимодействия дефекта, расположенным в начале координат, с возбуждением, такая, что при $U_{0}>0$ возбуждение отталкивается от дефекта, а при $U_{0}<0$ - притягивается.

Стационарные состояния с энергией $E$ уравнений (1) можно получить, представив в виде: $\psi(x, t)=\psi(x) e^{-i E t}$. Решение уравнения (1) сводится к решению двух стационарных УШ на полуосях:

$$
\begin{gathered}
\frac{1}{2 m} \psi^{\prime \prime}(x)+\left(E-\Omega_{1}\right) \psi(x)=0, x<0, \\
\frac{1}{2 m} \psi^{\prime \prime}(x)+\left(E-\Omega_{2}\right) \psi(x)+\gamma|\psi(x)|^{2} \psi(x)=0, \\
x>0,
\end{gathered}
$$

с граничными условиями:

$$
\begin{gathered}
\psi(-0)=\psi(+0) \\
\psi^{\prime}(+0)-\psi^{\prime}(-0)=2 m U_{0} \psi(0)
\end{gathered}
$$

В линейной среде без дефекта распространяются свободные волны с квадратичным законом дисперсии. Как известно, в такой среде симметричное локализованное по обе стороны от дефекта состояние с функцией $\psi(x)=A e^{-q|x|}$, где $q=-m U_{0}$, реализуется для притягивающего дефекта с $U_{0}<0$, а энергия локального уровня $E=\Omega-m U_{0}^{2} / 2$. В [6] отмечалось, что при наличии дефекта в линейной среде возможно существование локализованных состоянии с несимметричной частью волны, распространяющейся только по одну сторону от дефекта.

\section{2. НЕЛИНЕЙНАЯ СРЕДА С ПОЛОЖИТЕЛЬНЫМ АНГАРМОНИЗМОМ}

В отсутствие границы раздела в нелинейной среде при положительном ангармонизме (при $\gamma>0$ ) и одинаковых параметрах сред $\Omega_{\mathrm{j}}=\Omega, j=1,2$, распространяется свободный солитон:

$$
\psi(x)=\frac{A}{\operatorname{ch} q\left(x-x_{0}\right)},
$$

локализованный перпендикулярно направлению своего движения с максимумом в точке $x=x_{0}$, причем волновое число $q$ является свободным параметром, амплитуда локализации: $A=q(m \gamma)^{-1 / 2}$, энергия: $E=\Omega-q^{2} / 2 m$.

При наличии границы раздела линейной и нелинейной сред возможно существование локализованного несимметрично относительно дефекта состояния, описываемого решением уравнений(2) и (3), удовлетворяющим граничным условиям (4) и (5):

$$
\psi(x)=\left\{\begin{array}{l}
A e^{\kappa x}, x<0 \\
\frac{B}{\operatorname{ch} q\left(x-x_{0}\right)}, x>0
\end{array}\right.
$$

где $A=\frac{q}{(m \gamma)^{1 / 2} \operatorname{ch} q x_{0}}, B=q(m \gamma)^{-1 / 2}, \kappa^{2}=2 m\left(\Omega_{1}-E\right)$, $q^{2}=2 m\left(\Omega_{2}-E\right)$, а уровни энергии определяются из дисперсионного соотношения:

$$
q \text { th } q x_{0}-\kappa=2 m U_{0} .
$$

Такие состояния реализуются ниже сплошного спектра при $E<\min \left(\Omega_{1,2}\right)$. Если $\Omega_{\mathrm{j}}=\Omega$, то $q=\kappa$ из (8) получится выражение: $q\left(\right.$ th $\left.q x_{0}-1\right)=2 m U_{0}$.

Из дисперсионного соотношения (8) следует, что энергия локализованных состояний зависит от параметра $x_{0}$, значение которого может быть определено из условия нормировки волновой функции.

В [1] было рассмотрено локализованное вблизи поверхности состояние, описываемое функцией вида (7), отвечающее случаю отсутствия активного взаимодействия возбуждения с границей раздела линейной и нелинейной сред, когда $U_{0}=0$.

Внутри сплошного спектра в диапазоне $\Omega_{1}<E<\Omega_{2}$ возможно существование несимметричного состояния, локализованного только в нелинейной среде и описываемого решением уравнений (2) и (3), удовлетворяющим граничным условиям (4) и (5):

$$
\psi(x)= \begin{cases}A \cos (k x+\varphi), & x<0 \\ \frac{B}{\operatorname{ch} q\left(x-x_{0}\right)}, & x>0\end{cases}
$$


где в полупространстве с нелинейной средой параметры решения такие же, как и для (7), а в полупространстве с линейной средой теперь распространяется стоячая волна с волновым числом $k^{2}=2 m\left(E-\Omega_{1}\right)$ и амплитудой

$$
A=\frac{q \Delta_{c}}{k(m \gamma)^{1 / 2} \operatorname{ch} q x_{0}},
$$

где $d_{c}=q$ th $q x_{0}-2 m U_{0}, \Delta_{c}^{2}=k^{2}+d_{c}^{2}$. Уровни энергии, соответствующие состояниям (9), определяются из дисперсионного соотношения:

$$
\operatorname{tg} \varphi=d_{c} / k .
$$

Рассматриваемое состояние (9) описывает волну, распространяющуюся в полупространстве с линейной средой, которая при взаимодействии с границей раздела в полупространстве с нелинейной средой монотонно затухает при удалении от границы.

Можно отметить существование состояния с $\varphi=0$, спектр которого определятся из выражения: $q$ th $q x_{0}=2 m U_{0}$. Отсюда в явном виде можно получить значения энергии в двух предельных случая:

$$
E=\left\{\begin{array}{l}
\Omega_{2}-U_{0} / E_{0}, q x_{0} \ll 1 \\
\Omega_{2}-2 m U_{0}^{2}, q x_{0} \gg 1 .
\end{array}\right.
$$

Требование малости $q x_{0} \ll 1$ означает, что уровни энергии должны быть близки к краю зоны сплошного спектра: $\left|\Omega_{2}-E\right|<<1 / 2 m x_{0}^{2}$, причем для существования связанных состояний такого вида должны выполняться одни из требований: 1) $U_{0}>0$ и $x_{0}>0$ или 2) $U_{0}<0$ и $x_{0}<0$. Это означает, что локализованные в одном полупространстве состояния вида (9) существуют как для притягивающего, так и для отталкивающего дефекта.

Из (10) в таком пределе $q x_{0} \ll 1$ для произвольных $\varphi$ можно в явном виде получить энергию:

$$
E=\frac{2 m U_{0}\left(1-2 \Omega_{2} x_{0}\right)+\Omega_{1} \operatorname{tg}^{2} \varphi}{\operatorname{tg}^{2} \varphi-4 m U_{0} x_{0}} .
$$

Оказалось, что локализованное в одной части полупространства состояние, существует внутри сплошного спектра собственных стационарных состояний линейных волн. Такие состояния принято называть квазилокальными.Зависимость фазы от энергии $\varphi=\varphi(E)$ позволяет получить описанным в [7] способом добавку к плотности квазилокальных состояний:

$$
\delta g(E)=\frac{1}{\pi} \frac{\partial \varphi}{\partial E}
$$

Тогда из дисперсионного соотношения (10) для рассматриваемого квазилокального состояния:

$$
\delta g_{c}(E)=\delta g_{1 c}(E)+\delta g_{2 c}(E),
$$

где

$$
\begin{gathered}
\delta g_{1 c}(E)=-\frac{d_{c}}{\pi \Delta_{c}} \sqrt{\frac{m}{2\left(E-\Omega_{1}\right)}}, \\
\delta g_{2 c}(E)=-\frac{k d_{c}^{\prime}}{\pi \Delta_{c}} \sqrt{\frac{m}{2\left(\Omega_{2}-E\right)}}, \\
d_{c}^{\prime}=\text { th } q x_{0}+q x_{0} / \operatorname{ch}^{2} q x_{0} .
\end{gathered}
$$

Можно отметить, что если энергия удовлетворяет требованию $q$ th $q x_{0}<2 m U_{0}$, то $\delta g_{1 c}(E)>0$. Плотности состояний (14) имеет характерные для одномерных моделей корневые особенности на краях сплошного спектра. Полюсы плотности состояний (14) определяются из требования $\Delta_{c}=0$, реализация которого возможна только при комплексном волновом числе, то есть когда $k=i \kappa$, что приводит к дисперсионному соотношению (8) для локальных состояний вида (7).

\section{3. НЕЛИНЕЙНЫЕ СРЕДЫ С ОТРИЦАТЕЛЬНЫМ АНГАРМОНИЗМОМ}

Рассмотрим теперь случай отрицательного значения параметра нелинейности среды в полупространстве, когда $\gamma<0$ для всех $x>0$. В отсутствие дефекта в нелинейной среде с отрицательным ангармонизмом и одинаковых параметрах сред $\left(\Omega_{\mathrm{j}}=\Omega\right.$, $j=1,2$ ) возможно существование неограниченного решения НУШ вида $\psi(x)=A / \operatorname{sh} q\left(x-x_{0}\right)$, с энергией $E=\Omega-q^{2} / 2 m$ и амплитудой $A=q / \sqrt{g m}$, $g=-\gamma>0$.

При наличии границы раздела линейной и нелинейной сред возможно существование локализованного несимметрично относительно дефекта состояния ниже сплошного спектра при $E<\min \left(\Omega_{1,2}\right)$, описываемого решением уравнений (2) и (3), удовлетворяющим граничным условиям (4) и (5):

$$
\psi(x)=\left\{\begin{array}{l}
A e^{\mathrm{K} x}, x<0 \\
\frac{B}{\operatorname{sh} q\left(x-x_{0}\right)}, x>0
\end{array}\right.
$$

где $A=q /(m g)^{1 / 2} \operatorname{sh} q x_{0}, B=q(m g)^{-1 / 2}$, а уровни энергии определяются из дисперсионного соотношения:

$$
q \text { cth } q x_{0}-\kappa=2 m U_{0} .
$$

При выполнении требования $x_{0}<0$ решение (16) всюду ограничено и удовлетворяет условию на бесконечности $|\psi(x)| \rightarrow 0$.

Если считать сначала, что $\Omega_{1}<\Omega_{2}$, то внутри сплошного спектра в диапазоне $\Omega_{1}<E<\Omega_{2}$ возможно существование несимметричного состоя- 


\section{C. Е. САВОТЧЕНКО}

ния, локализованного только в нелинейной среде и описываемого решением уравнений (2) и (3), удовлетворяющим граничным условиям (4) и (5):

$$
\psi(x)=\left\{\begin{array}{l}
A \cos (k x+\phi), x<0 \\
\frac{B}{\operatorname{sh} q\left(x-x_{0}\right)}, x>0
\end{array}\right.
$$

где в полупространстве с нелинейной средой параметры решения такие же, как и для (16), а в полупространстве с линейной средой теперь распространяется стоячая волна с волновым числом, как и для (9), и амплитудой

$$
A=-\frac{q \Delta_{s}}{k(m g)^{1 / 2} \operatorname{sh} q x_{0}},
$$

где $d_{s}=q \operatorname{cth} q x_{0}-2 m U_{0}, \Delta_{s}^{2}=k^{2}+d_{s}^{2}$. Уровни энергии, соответствующие состояниям (19), определяются из дисперсионного соотношения:

$$
\operatorname{tg} \varphi=d_{s} / k \text {. }
$$

Можно отметить существование состояния с $\varphi=0$, спектр которого определятся из выражения: $q$ cth $q x_{0}=2 m U_{0}$. Отсюда в явном виде можно получить значения энергии в двух предельных случая:

$$
E=\left\{\begin{array}{l}
\Omega_{2}-\frac{3}{x_{0}}\left(U_{0}-\frac{1}{2 m E_{0}}\right), q x_{0} \ll 1 \\
\Omega_{2}-2 m U_{0}^{2}, q x_{0} \gg 1 .
\end{array}\right.
$$

Из (20) в пределе $q x_{0}<<1$ для произвольных $\varphi$ можно в явном виде получить энергию:

$$
E=\Omega_{1}+\frac{1}{2 m \operatorname{tg}^{2} \phi}\left(\frac{1}{x_{0}^{2}}-\frac{2 m U_{0}}{x_{0}}+4 m^{2} U_{0}^{2}\right)
$$

Из (11) и (21) видно, что при больших энергиях уровни как для случая положительной, так и отрицательной нелинейности среды, асимптотически одинаковы.

Воспользовавшись дисперсионным соотношением (20), по формуле (13) можно получить добавку к плотности квазилокальных состояний рассматриваемого типа:

$$
\delta g_{s}(E)=\delta g_{1 s}(E)+\delta g_{2 s}(E)
$$

где

$$
\begin{gathered}
\delta g_{1 s}(E)=-\frac{d_{s}}{\pi \Delta_{s}} \sqrt{\frac{m}{2\left(E-\Omega_{1}\right)}}, \\
\delta g_{2 s}(E)=-\frac{k d_{s}^{\prime}}{\pi \Delta_{s}} \sqrt{\frac{m}{2\left(\Omega_{2}-E\right)}}, \\
d_{s}^{\prime}=\operatorname{cth} q x_{0}-q x_{0} / \mathrm{sh}^{2} q x_{0} .
\end{gathered}
$$

Следует отметить, что $\delta g_{1 s}(E)>0$, когда энергия удовлетворяет требованию $q \mathrm{cth} q x_{0}<2 m U_{0}$.
Поскольку для состояния вида (18) должно быть $x_{0}<0$, то выполнение указанного требования возможно при $U_{0}<0$, то есть для притягивающего дефекта.

Плотности состояний (23), также как (14), имеет характерные для одномерных моделей корневые особенности на краях сплошного спектра. Полюсы плотности состояний (23) определяются из требования $\Delta_{s}=0$, реализация которого возможна только при комплексном волновом числе, то есть когда $k=i \kappa$, что приводит к дисперсионному соотношению (17) для локальных состояний вида (16).

Известно, что в идеальной среде с отрицательной нелинейностью, когда $\gamma<0$, при $E>\Omega$ возможно существование всюду ограниченного решения НУШ в виде кинка $\psi(x)=A$ th $q_{t}\left(x-x_{0}\right)$, с энергией $E=q_{t}^{2} / m-\Omega$ и амплитудой $A= \pm q_{t} / \sqrt{g m}$.

Если теперь считать, что $\Omega_{2}<\Omega_{1}$, то в диапазоне $\Omega_{2}<E<\Omega_{1}$ локализованное состояние будет описываться решением

$$
\psi(x)=\left\{\begin{array}{l}
A e^{\kappa x}, x<0 \\
B \text { th } q_{t}\left(x-x_{0}\right), x>0
\end{array}\right.
$$

где $A=\mp q_{t}$ th $q x_{0}(m g)^{-1 / 2}, B= \pm q_{t}(m g)^{-1 / 2}$, а уровни энергии определяются из дисперсионного соотношения:

$$
2 q_{t} / \operatorname{sh} 2 q x_{0}+\kappa=-2 m U_{0} .
$$

Из (26) в предельном случае $q_{t} x_{0}<<1$ можно выразить энергию в явном виде:

$$
E=\Omega_{1}-\left(2 m U_{0}+1 / x_{0}\right)^{2} .
$$

В диапазоне $E>\max \left(\Omega_{1,2}\right)$ существует квазилокальное состояние, описываемое решением вида:

$$
\psi(x)=\left\{\begin{array}{l}
A \cos (k x+\varphi), x<0 \\
B \operatorname{th} q_{t}\left(x-x_{0}\right), x>0
\end{array}\right.
$$

где параметры решения в полупространстве с нелинейной средой такие же, как и для (25), в полупространстве с линейной средой волновое число $k$ такое же, как и для (9), а амплитуда

$$
A=\mp \frac{q \cos \varphi}{(m g)^{1 / 2} \text { th } q x_{0}} .
$$

Уровни энергии, соответствующие состояниям (28), определяются из дисперсионного соотношения:

$$
\operatorname{tg} \varphi=\Delta_{t 1} / \Delta_{t 2}
$$

где $\Delta_{t 1}=2\left(m U_{0} \operatorname{sh} 2 q_{t} x_{0}+q_{t}\right), \Delta_{t 2}=k \operatorname{sh} 2 q_{t} x_{0}$.

Из (29) в пределе $q x_{0}<<1$ можно в явном виде получить энергию:

$$
E=\frac{\left(2 m U_{0}+1 / x_{0}\right)^{2}}{2 m \operatorname{tg}^{2} \varphi} .
$$


В частном случае при $\Omega_{1,2}=0$ будет $k=\sqrt{2} q_{t}$, и тогда, воспользовавшись дисперсионным соотношением (29), по формуле (13) можно получить добавку к плотности квазилокальных состояний рассматриваемого типа:

$$
\delta g_{s}(E)=\frac{1}{2 \pi} \sqrt{\frac{m}{E}} \frac{\Delta_{t 1}^{\prime} \Delta_{t 2}-\Delta_{t 1} \Delta_{t 2}^{\prime}}{\Delta_{t 1}^{2}+\Delta_{t 2}^{2}}
$$

где $\Delta_{t 1}^{\prime}=2\left(2 m U_{0} x_{0} \operatorname{ch} 2 q_{t} x_{0}+1\right)$, $\Delta_{t 2}^{\prime}=\sqrt{2}\left(\operatorname{sh} 2 q_{t} x_{0}+2 q_{t} x_{0} \operatorname{ch} 2 q_{t} x_{0}\right)$.

\section{ЗАКЛЮЧЕНИЕ}

В работе получены решения НУШ, описывающие взаимодействие возбуждений на границы раздела линейной и нелинейной сред. В зависимости от соотношения энергии возбуждения и значений краев зоны сплошного спектра собственных стационарных линейных состояний возникают как локализованные по обе стороны от границы раздела сред состояния, так и квазилокальные состояния. Возбуждение с энергией в полосе сплошного спектра линейных волн представляет собой стоячую волну в линейной среде и затухающую при удалении от границы раздела в нелинейной среде. В частных предельных случаях получены энергетические уровни стационарных квазилокальных состояний в явном виде.

Проанализированы случаи нелинейных сред с положительным и отрицательным ангармонизмами межатомного взаимодействия. В случае среды с отрицательной нелинейностью существуют два вида локализованных состояний с отличающимися частными решениями НУШ в полупространстве с нелинейной средой в различных диапазонах энергии.

Для квазилокальных состояний получены добавки к спектральной плотности. Полюсы спектральной плотности определяют спектр локализованных состояний.

Следует отметить, что в рассматриваемой системе, помимо солитонных, возможны периодические решения НУШ, описывающие трансформацию кноидальных волн при переходе через границу раздела линейной и нелинейной сред. Однако детальный анализ таких эффектов требует отдельного рассмотрения.

\section{СПИСОК ЛИТЕРАТУРЫ}

1. Ахмедиев Н. Н., Корнеев В. И., Кузьменко Ю. В. // ЖЭТФ, 1985, т. 88, № 1, с. 107-115.

2. Герасимчук И. В., Ковалев А. С. // Физика низких mемператур, 2000, т. 26, № 8, с. 799-809.

3. Abdullaev F. Kh., Baizakov B. B., Umarov B. A. // Optics Communications, 1998, vol. 156, pp. 341-346.

4. Савотченко С. Е. // Известия высших учебных заведений. Физика, 2004, т. 47, № 5, с. 79-84.

5. Савотченко С. Е. // Вестник Воронежского государственного университета. Серия: Физика. Математика, 2016, № 4, с. 51-59. 317.

6. Косевич А. М. // ЖЭТФ, 1999, т. 115, № 1, с. 306-

7. Косевич А. М., Мацокин Д. В., Савотченко С. Е. // Письма в ЖЭТФ, 2001, т. 73, № 11-12, с. 680-683.

\title{
LOCALIZED STATES ON THE BOUNDARY BETWEEN LINEAR AND NONLINEAR MEDIA
}

\author{
(C) 2017 S. E. Savotchenko \\ Belgorod State Technological University named after V. G. Shukhov, \\ 46 Kostyukova str., 308012 Belgorod, Russia \\ e-mail:savotchenkose@mail.ru
}

Received 02.10.2017

\begin{abstract}
The paper presents a model describing the peculiarities of excitation localization on the boundary between linear and nonlinear media. The boundary of nonlinear media, characterised by various parameters of anharmonicity of the interatomic interaction, creates a disturbance of the medium characteristics, which is located at distances much smaller than the width of the localization of propagating waves. The model is based on linear and nonlinear Schrцdinger equations on halfspaces.Explicit solutions of nonlinear Schrцdinger equations satisfying the boundary conditions were found for positive and negative nonlinearity parameters.It was demonstrated that nonlinear localized excitations can be of several types. The structure and the shape of the localized states are determined by the type of anharmonicity of the interaction in the medium and the intensity of the interaction
\end{abstract}




\section{C. Е. САВОТЧЕНКО}

between the excitations and the defect.There are two types of states: localized and quasilocal.Localized states are described by wave functions that damp away from the interface of media under various laws. The profile of such a bound state is asymmetric in relation to the media interface.Quasi-local states are described by wave functions monotonically damped in a half-space with a nonlinear medium and a standing wave in a half-space with a linear medium. The wave is localized during the transition through the medium interface. The dispersion relations determining the values of the energy of localized and quasilocal states were obtained.The dependence of the wave numbers on the parameters of the system for localized states in various limiting cases was demonstrated.The additions to the spectral density of quasilocal states were derived.

Keywords: nonlinear Schrцdinger equation, boundary media, soliton, localized states, quasi-localized, density of states.

\section{REFERENCES}

1. Ahmediev N. N., Korneev V. I., Kuzmenko U. V. Sov. Phys. JETP, 1985, vol. 61, no. 1, pp. 62-67. Available at: http://www.jetp.ac.ru/cgi-bin/dn/e_061_01_0062.pdf

2. Gerasimchuk I. V., Kovalev A. S. Low Temp. Phys., 2000, vol. 26, no. 8, pp. 799-809.

3. Abdullaev F. Kh., Baizakov B. B., Umarov B. A. Optics Communications, 1998, vol. 156, pp. 341-346. https://doi.org/10.1016/S0030-4018(98)00451-9

4. Savotchenko S. E. Russian Physics Journal, 2004, vol. 47, no. 5, pp. 556-562. Available at: https://link.springer. com/content/pdf/10.1023\%2FB\%3ARUPJ.00000463 30.92744.73.pdf
5. Savotchenko S. E. Proceedings of Voronezh State University. Series: Physics. Mathematics, 2016, no. 4, pp. 51-59. Available at: http://www.vestnik.vsu.ru/pdf/ physmath/2016/04/2016-04-05.pdf (in Russian)

6. Kosevich A. M. JETP, 1999, vol. 88, no. 1, pp. 168 173. Available at: https://link.springer.com/content/pdf/ 10.1134\%2F1.558779.pdf

7. Kosevich A. M. Matsokin D. V., Savotchenko S. E. JETP Letters, 2001, vol. 73, no. 11, pp. 600-603. Available at: https://link.springer.com/content/pdf/10.1134\%2F1. 1392420.pdf
Савотченко Сергей Евгеньевич - д. ф. - м. н, доцент, профессор кафедры высшей математики, Белгородский государственный технологический университет имени В. Г. Шухова; тел.: +7(920) 5610446, e-mail: savotchenkose@mail.ru
Savotchenko Sergey E. - Dr. Sci. (Phys.-Math.), Associate Professor, Professor of High Mathematics Department, Belgorod State Technological University named after V. G. Shukhov; ph.: +7(920)5610446, e-mail: savotchenkose@mail.ru 